\section{JAK Inhibition as a Therapeutic Strategy for IgG4-RD}

Khan $\mathrm{S}^{1}$, Gordins $\mathrm{P}^{1}$, Durairaj $\mathrm{S}^{2}$

${ }^{1}$ Department of Immunology \& Allergy, Castle Hill Hospital, Cottingham, UK

${ }^{2}$ Department of Haemato-Oncology, Castle Hill Hospital, Cottingham, UK

J Investig Allergol Clin Immunol 2021; Vol. 31(3): 280-281 doi: 10.18176/jiaci.0654

Key words: IgG4-related disease. IgG4-RD. Fibrosis. Cytokines. JAK inhibition.

Palabras clave: Enfermedad relacionada con IgG4. ER-IgG4. Fibrosis. Citoquinas. Inhibición de las JAK.

\section{To the Editor:}

The review by Carballo et al [1] provides an excellent summary of the varied clinical presentations of IgG4-related disease (IgG4-RD), highlighting the role of clinicians and insisting on a detailed pathological description of affected organs with IgG4-stained plasmablasts infiltrating tissues to arrive at a diagnosis. However, the cytokine environment and what makes this "nonpathogenic" IgG isotype cause irreversible end-organ damage remains unknown. As the authors rightly discuss, both autoimmune and allergic aspects of IgG4-RD are involved. In addition to the general approach to managing the diverse clinical presentations of this disease, we would like to discuss the cytokine signalling involved in IgG4-RD in more detail and suggest Janus-associated kinase (JAK) inhibition as alternative therapeutic strategy to manage this condition.

Autoimmunity implies a perpetual cycle that is initiated by a cell-damaging event that modifies self-antigens. Tissueresident antigen-presenting cells increase MHC class II expression and present modified antigens to $\mathrm{T}$ cells, and the unique cytokine environment switches $\mathrm{CD}^{+} \mathrm{T}_{\mathrm{H}} 2 \mathrm{~T}$-cell differentiation, which in turn drives an adaptive immune response towards IgG4 plasma cell differentiation and, eventually, end-organ damage with fibrosis (Figure).

The pathogenic role of IgG4 was shown to involve passive transfer of antibodies from patients with IgG4-RD causing pancreatic and salivary gland injury in neonatal $\mathrm{Balb} / \mathrm{c}$ mice [2]. Distinct glycosylation changes on $\mathrm{IgG} 4$ with increased G0 and F1 glycans in patients with IgG4-RD and hypocomplementemia suggest activation of the lectin pathway on phagocytes to induce chronic inflammation $[3,4]$. Interestingly, in the pancreatic ovalbumin mouse model

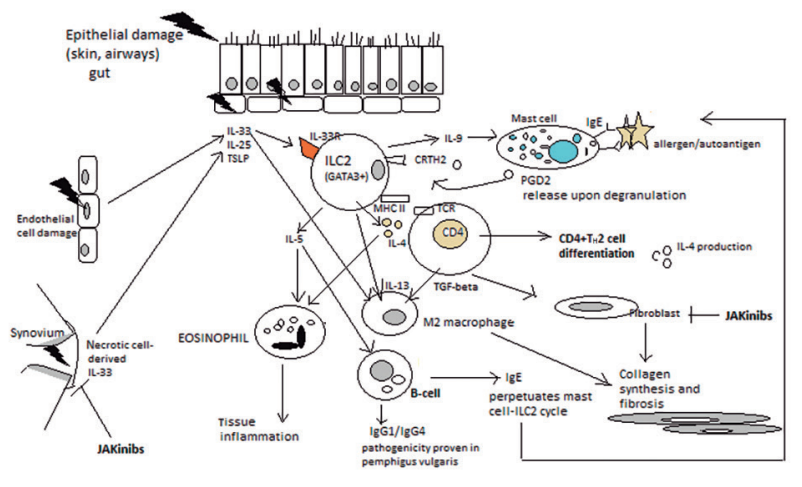

Figure. Schematic representation of pathways involving innate lymphoid cells type 2 (ILC2) and cytokines in IgG4-related disease. Epithelial damage leads to upregulation of IL-33, IL-25, and TSLP, with IL-33 acting via ST2 (IL-33R) on ILC2 cells and cytokines such as IL-4, IL-5 and IL-13 leading to eosinophil recruitment and macrophage polarization. MHC class II upregulation on ILC2 interacts with TCR on CD4+ T cells (also CD80/86 and CD28; OX40L and OX40) to further produce IL-4. Upregulation of GATA3 upregulation leads to $\mathrm{CD}^{+} \mathrm{T}_{\mathrm{H}} 2$ cell differentiation. $\mathrm{CD}^{+}$cytotoxic lymphocytes release TGF- $\beta$ that activate fibroblasts, including M2 macrophages, leading to collagen synthesis and fibrosis (CD206+ subset associated with fibrosis in systemic sclerosis). IL-5 can induce class-switching of B cells to produce $\lg \mathrm{G} 1 / \operatorname{lgG} 4$ or $\lg$ E that binds to mast cells. Upon further (auto)antigen cross-linking, the mast cells degranulate, releasing PGD2 that interacts with CRTH2 on ILC2s, thus perpetuating the cycle of cytokine release. Endothelial damage results in production of IL-4, IL-12, TGF- $\beta$, IL-6, and IL-33 that leads to proliferation of fibroblasts and polarization of macrophages with survival signals for differentiation into myofibroblasts and production of extracellular matrix. Necrotic cells from inflamed joints and fibroblast-like synoviocytes release IL-33, which expands synovial-resident ILCs. Further feedback via arthritogenic TH17 cells amplify and lead to chronic joint inflammation.

(RIP-mOVA mice), no tissue inflammation was observed when animals were exposed to recombinant ovalbuminspecific human IgG4 monoclonal antibody only in contrast to cotransfer of OVA-specific $\mathrm{CD} 8^{+}$cytotoxic $\mathrm{T}$ cells, which resulted in significant tissue damage, thus suggesting the crucial role of $\mathrm{T}$ cells in the pathogenesis of IgG4-RD [5]. It is uncertain whether IgG1 antibodies confer pathogenicity along with $\operatorname{IgG} 4$, especially with identification of annexin A11-specific IgG1 and IgG4 antibodies in patients with IgG4$\mathrm{RD}$ affecting the biliary tract, salivary gland, or pancreas [6].

As the pathologic process in IgG4-RD can involve almost any tissue in the body, it is likely that the sustained cellular response is due to a ubiquitous cytokine signal(s). In this context, it is worthwhile noting that all cells in the body have the ability to respond to IL-4 and IL-13 cytokines, including astrocytes and microglial cells, which are macrophage-like cells in the central nervous system, thus perhaps providing an explanation for leptomeningeal IgG4-RD. Tsuboi et al [7] showed that patients with Sjögren syndrome differ from those with IgG4-RD sialadenitis, in which IL-10 and TGF- $\beta$ were 
significantly elevated. The pleiotropic effects of IL-4 and IL13 produced by $\mathrm{CD} 4+$ in variant natural killer (iNKT) cells and/or group 2 innate lymphoid cells (ILC2) in IgG4-RD, as well as signalling through type 1 (for lymphocytes) and type 2 (for epithelial cells) IL-4 receptors via JAK1/JAK3 (IL-4) or Tyk2/JAK3 (IL-13) with downstream STAT6, are likely to drive chronic tissue inflammation and fibrosis [8] (Figure).

This cytokine model of self-sustained signalling implies that JAK inhibitors (small molecules that inhibit JAK1, JAK2, JAK3, and Tyk2) may be useful in controlling tissue inflammation and preventing fibrosis in patients with IgG4-RD and may prove to be as promising as the findings from recent clinical trials in several other autoimmune diseases $[8,9]$. When cocultured with tofacitinib (first-generation JAK1/3 inhibitor with some anti-JAK2 activity), synovial fibroblasts lost their ability to migrate to form networks and down-regulate production of inflammatory cytokines and metalloproteinases. Tofacitinib prevented bleomycin-induced skin and lung fibrosis in mice, including reduction of skin fibrosis in tight skin 1 (TSK1/+) mice, which is a model for the human fibrotic skin disorder scleroderma. It was also able to reverse graft-versushost disease and provide endothelial cell protection, thus indicating its multiple effects on lowering tissue inflammation. Tofacitinib $5 \mathrm{mg}$ twice daily was also effective in moderateto-severe rheumatoid arthritis and psoriatic arthritis, with an overall satisfactory safety profile and only a small increase in the frequency of malignancies and serious infections. Baricitinib (JAK1/2 inhibitor), which inhibits expression of costimulating molecules CD80/CD86 on monocyte-derived dendritic cells and production of type 1 interferons by plasmacytoid dendritic cells, including production of IL-6 and differentiation of $\mathrm{B}$ cells into plasmablasts, may be an ideal candidate for managing IgG4-RD. It was effective in anti-TNF inhibitor-refractory rheumatoid arthritis, although it increased the risk of thromboembolic events. Ruxolitinib (JAK2/1 inhibitor), tofacitinib, and itacitinib (selective JAK1 inhibitor) decreased M2 macrophage activation by inhibiting IL-4 and IL-13 signalling and improved skin and pulmonary inflammation in a mouse model that mimics sclerodermaassociated interstitial lung disease [10]. JAK inhibitors may therefore have a significant role to play in IgG4-RD. However, physicians prescribing these drugs will need to be mindful of the risks of novel infections, as well as the possible reemergence of old infections.

\section{Funding}

The authors declare that no funding was received for the present study.

\section{Conflicts of Interest}

The authors declare that they have no conflicts of interest.

\section{References}

1. Carballo I, González-Quintela A, Sopeña B, Vidal C. Immunoglobulin G4-related Disease: What an Allergist Should Know. J Investig Allergol Clin Immunol. 2021 [Ahead of print].

2. Shiokawa M, Kodama Y, Kuriyama K, Yoshimura K, Tomono T, Morita $T$, et al. Pathogenicity of $\mathrm{lgG}$ in patients with lgG4related disease. Gut. 2016;65:1322-32.

3. Culver EL, van de Bovenkamp FS, Derksen NIL, Koers J, Cargill T, Barnes E, et al. Unique patterns of glycosylation in immunoglobulin subclass G4-related disease and primary sclerosing cholangitis. J Gastroenterol Hepatol. 2019;34:187886.

4. Konno N, Sugimoto M, Takagi T, Furuya M, Asano T, Sato S, et al. Changes in N-glycans of IgG4 and its relationship with the existence of hypocomplementemia and individual organ involvement in patients with lgG4-related disease. PLoS One. 2018;13:e0196163.

5. Sasaki T, Yajima T, Shimaoka T, Ogawa S, Saito T, Yamaoka $K$, et al. Synergistic effect of IgG4 antibody and CTLs causes tissue inflammation in IgG4-related disease. Int Immunol. 2020;32:163-74.

6. Hubers LM, Vos $H$, Schuurman AR, Erken R, Oude Elferink RP, Burgering $B$, et al. Annexin A11 is targeted by $\lg G 4$ and $\lg G 1$ autoantibodies in IgG4-related disease. Gut. 2018;67:72835.

7. Tsuboi H, Matsuo N, lizuka M, Tsuzuki S, Kondo Y, Tanaka A, et al. Analysis of lgG4 class switch-related molecules in lgG4related disease. Arthritis Res Ther. 2012;14:R171.

8. Schwartz DM, Kanno Y, Villarino A, Ward M, Gadina M, O'Shea JJ. JAK inhibition as a therapeutic strategy for immune and inflammatory diseases. Nat Rev Drug Discov. 2017;17:78.

9. Fragoulis GE, McInnes IB, Siebert S. JAK-inhibitors. New players in the field of immune-mediated diseases, beyond rheumatoid arthritis. Rheumatology (Oxford). 2019;58:i43-i54.

10. Lescoat A, Lelong M, Jeljeli M, Piquet-Pellorce C, Morzadec C, Ballerie $A$, et al. Combined anti-fibrotic and anti-inflammatory properties of JAK-inhibitors on macrophages in vitro and in vivo: Perspectives for scleroderma-associated interstitial lung disease. Biochem Pharmacol. 2020;178:114103.

Manuscript received November 17, 2020; accepted for publication November 20, 2020.

\section{Sujoy Khan}

\author{
Department of Immunology \& Allergy \\ Castle Hill Hospital \\ Cottingham, HU16 5JQ, UK \\ E-mail: sujoykhan@gmail.com
}

